\title{
Two Simplified Recursive Gauss-Newton Algorithms for Direct Amplitude and Phase Tracking of a Real Sinusoid
}

\author{
Jun Zheng, Kenneth W. K. Lui, W.-K. Ma, and H. C. So
}

\begin{abstract}
In this letter, the problem of adaptive tracking the amplitude and phase of a noisy sinusoid with known frequency is addressed. Based on approximating the recursive Gauss-Newton approach, two computationally simple algorithms, which provide direct parameter estimates, are devised and analyzed. Simulation results show that the proposed methods can attain identical estimation performance as their original one.
\end{abstract}

Index Terms-Fast algorithm, Gauss-Newton method, sinusoidal amplitude estimation, sinusoidal phase estimation.

\section{INTRODUCTION}

@INUSOIDAL parameter estimation from noisy measurements has many important applications in sonar, radar, digital communications, and biomedical engineering [1]-[5]. In this letter, we address the problem of amplitude and phase tracking for a single real sinusoid with known frequency. The discrete-time signal model is

$$
x(n)=A(n) \sin (\omega n+\phi(n))+v(n)
$$

where $\omega$ is the known frequency, $A(n)>0$ and $\phi(n) \in[-\pi, \pi)$, which can be time-varying, represent the amplitude and phase of the sinusoid at time $n$, respectively, and $v(n)$ is the additive zero-mean white noise with variance $\sigma^{2}$.

Since $x(n)$ is a nonlinear function of the parameters of interest, one standard technique [1] is to make the expression linear by transforming $A(n)$ and $\phi(n)$ into $A(n) \cos (\phi(n))$ and $A(n) \sin (\phi(n))$. We can then apply the recursive least squares (RLS) or recursive Gauss-Newton (RGN) method [6] to solve the problem, but the computational complexity of both schemes is high. In this letter, we utilize the RGN approach to develop two computationally simple algorithms for directly tracking $A(n)$ and $\phi(n)$. Following the Gauss-Newton method for maximum-likelihood estimation of the amplitude, frequency, and phase for multiple sinusoids [3], as well as the RGN framework [6], the RGN algorithm for direct amplitude and phase estimation is presented in Section II. By exploiting the characteristics of the Hessian matrix in the RGN algorithm,

\footnotetext{
Manuscript received April 3, 2007; revised July 10, 2007. This work was supported by a grant from CityU (Project No. 7001924). The associate editor coordinating the review of this manuscript and approving it for publication was Dr. Patrick A. Naylor.

J. Zheng, K. W. K. Lui, and H. C. So are with the Department of Electronic Engineering, City University of Hong Kong, Hong Kong (e-mail: junzheng@cityu.edu.hk; 50469990@student.cityu.edu.hk; hcso@ee.cityu.edu. hk).

W.-K. Ma is with the Department of Electrical Engineering and Institute of Communications Engineering, National Tsing Hua University, Taiwan, R.O.C. (e-mail: wkma@ieee.org).

Digital Object Identifier 10.1109/LSP.2007.906217
}

two simplified sinusoidal parameter estimators are devised and analyzed in Section III. Simulation results are included to evaluate the performance of the proposed algorithms in Section IV, and finally, conclusions are drawn in Section V.

\section{Recursive Gauss-Newton Method}

Define $\boldsymbol{\theta}(n)=[A(n), \phi(n)]^{T}$, where $T$ denotes the transpose, and let its estimate be $\hat{\boldsymbol{\theta}}(n)=[\hat{A}(n), \hat{\phi}(n)]^{T}$. Using $\hat{\boldsymbol{\theta}}(n-1)$, the estimate of $x(n)$ at time $n$ is computed as $\hat{A}(n-1) \sin (\omega n+\hat{\phi}(n-1))$. The a priori estimation error at time $n$ is then

$$
e(n)=x(n)-\hat{A}(n-1) \sin (\omega n+\hat{\phi}(n-1)) .
$$

A standard approach for tracking $\boldsymbol{\theta}(n)$ is to minimize the following exponentially weighted cost function:

$$
\mathcal{E}(n)=\sum_{i=0}^{n} \lambda^{n-i} e^{2}(i)
$$

where $0<\lambda<1$ is the forgetting factor. As (2) is not linear in $\hat{A}(n)$ and $\hat{\phi}(n)$, the conventional RLS algorithm cannot be applied for this problem. Here, we utilize a generalized form, the RGN algorithm, to minimize (3).

Following the Gauss-Newton method for sinusoidal parameter estimation [3] and the RGN algorithm derivation [6], the RGN updating equations for tracking $\boldsymbol{\theta}$ are easily shown to be

$$
\hat{\boldsymbol{\theta}}(n)=\hat{\theta}(n-1)-\mathbf{H}^{-1}(n) \boldsymbol{\psi}(n) e(n)
$$

and

$$
\mathbf{H}(n)=\lambda \mathbf{H}(n-1)+\boldsymbol{\psi}(n) \boldsymbol{\psi}^{T}(n)
$$

where (6) and (7) at the bottom of the next page are the gradient vector and the Hessian matrix, respectively. In practical implementation, the matrix inversion of $\mathbf{H}(n)$ in (4) is avoided, and we make use of the matrix inversion lemma to directly update $\mathbf{H}^{-1}(n)$ in (8) at the bottom of the next page instead of employing (5). To summarize, the RGN estimate of $\boldsymbol{\theta}$ is computed using (2), (4), (6), and (8).

\section{SimPLIFIED RECURSIVE ESTIMATION ALGORITHMS}

In spite of avoiding matrix inversion computation, the RGN method is still computationally demanding. In this section, we propose two simplified RGN estimators for $\boldsymbol{\theta}$ such that their computational complexity is significantly smaller without undermining the estimation performance, compared to the original one. 


\section{A. Hessian Matrix Approximation}

Here we approximate the Hessian matrix in (7). Since $\lambda$ is usually close to 1 and assuming that $\omega$ is not near 0 or $\pi$, we have [3]

$$
\begin{aligned}
\mathbf{H}(n) & \approx \sum_{i=0}^{n} \lambda^{n-i}\left[\begin{array}{cc}
1 / 2 & 0 \\
0 & \hat{A}^{2}(n-1) / 2
\end{array}\right] \\
& =\frac{1-\lambda^{n+1}}{2(1-\lambda)}\left[\begin{array}{cc}
1 & 0 \\
0 & \hat{A}^{2}(n-1)
\end{array}\right] .
\end{aligned}
$$

It is noteworthy that when the sinusoidal frequency is close to 0 or $\pi$, this approximation for $\mathbf{H}(n)$ becomes invalid, and thus, utilizing (9) will lead to a biased estimate of $\boldsymbol{\theta}$. Based on (9), $\mathbf{H}^{-1}(n)$ can be easily computed from

$$
\mathbf{H}^{-1}(n)=\left[\begin{array}{cc}
1 / c(n) & 0 \\
0 & 1 /\left[\hat{A}^{2}(n-1) c(n)\right]
\end{array}\right]
$$

where $c(n)=\left(1-\lambda^{n+1}\right) /[2(1-\lambda)]$. Substituting (10) into (4) and noting that $c(n)$ can be computed recursively, we have

$$
\begin{aligned}
\hat{A}(n)= & \hat{A}(n-1) \\
& +\sin (\omega n+\hat{\phi}(n-1)) e(n) / c(n) \\
\hat{\phi}(n)= & \hat{\phi}(n-1) \\
& +\cos (\omega n+\hat{\phi}(n-1)) \times e(n) /[\hat{A}(n-1) c(n)]
\end{aligned}
$$

and

$$
c(n)=\lambda c(n-1)+1 / 2 .
$$

We refer the updating equations of (2) and (11)-(13) to as the Hessian matrix approximation (HMA) algorithm.

\section{B. Gradient Correlation Matrix Approximation}

Based on (9), we can ignore the off-diagonal elements of $\mathbf{H}(n)$ in an alternative manner to achieve algorithm simplification. Here, we set the two off-diagonal elements in the gradient correlation matrix, namely, $\boldsymbol{\psi}(n) \boldsymbol{\psi}^{T}(n)$ of (5), to zero. As a result, (5) becomes (14) at the bottom of the page, where

$$
\begin{aligned}
h_{11}(n)= & \lambda h_{11}(n-1) \\
& +\sin ^{2}(\omega n+\hat{\phi}(n-1))
\end{aligned}
$$

and

$$
\begin{aligned}
h_{22}(n)= & \lambda h_{22}(n-1)+\hat{A}^{2}(n-1) \\
& \times \cos ^{2}(\omega n+\hat{\phi}(n-1)) .
\end{aligned}
$$

Putting (14) into (4) yields

$$
\begin{aligned}
\hat{A}(n)= & \hat{A}(n-1) \\
& +\sin (\omega n+\hat{\phi}(n-1)) \times e(n) / h_{11}(n)
\end{aligned}
$$

and

$$
\begin{aligned}
\hat{\phi}(n)= & \hat{\phi}(n-1)+\hat{A}(n-1) \\
& \times \cos (\omega n+\hat{\phi}(n-1)) e(n) / h_{22}(n) .
\end{aligned}
$$

We refer the updating equations of (2) and (15)-(18) to as the gradient correlation matrix approximation (GCMA) algorithm.

\section{Comparative Computational Complexity}

Considering that the cosine and sine functions are determined from lookup tables, the computational requirements for the

$$
\begin{aligned}
\boldsymbol{\psi}(i) & =\frac{\partial e(i)}{\partial \hat{\boldsymbol{\theta}}}=\left[\begin{array}{c}
-\sin (\omega i+\hat{\phi}(n-1)) \\
-\hat{A}(n-1) \cos (\omega i+\hat{\phi}(n-1))
\end{array}\right] \\
\mathbf{H}(n) & =\sum_{i=0}^{n} \lambda^{n-i} \boldsymbol{\psi}(i) \boldsymbol{\psi}^{T}(i) \\
& =\sum_{i=0}^{n} \lambda^{n-i}\left[\begin{array}{cc}
\sin ^{2}(\omega i+\hat{\phi}(n-1)) & \hat{A}(n-1) \sin (2(\omega i+\hat{\phi}(n-1))) / 2 \\
\hat{A}(n-1) \sin (2(\omega i+\hat{\phi}(n-1))) / 2 & \hat{A}^{2}(n-1) \cos ^{2}(\omega i+\hat{\phi}(n-1))
\end{array}\right]
\end{aligned}
$$

$$
\mathbf{H}^{-1}(n)=\frac{1}{\lambda}\left[\mathbf{H}^{-1}(n-1)-\frac{\mathbf{H}^{-1}(n-1) \boldsymbol{\psi}(n) \boldsymbol{\psi}^{T}(n) \mathbf{H}^{-1}(n-1)}{\lambda+\boldsymbol{\psi}^{T}(n) \mathbf{H}^{-1}(n-1) \boldsymbol{\psi}(n)}\right]
$$

$$
\begin{aligned}
\mathbf{H}(n) & \approx \lambda \mathbf{H}(n-1)+\left[\begin{array}{cc}
\sin ^{2}(\omega n+\hat{\phi}(n-1)) & 0 \\
0 & \hat{A}^{2}(n-1) \cos ^{2}(\omega n+\hat{\phi}(n-1))
\end{array}\right] \\
& =\left[\begin{array}{cc}
h_{11}(n) & 0 \\
0 & h_{22}(n)
\end{array}\right]
\end{aligned}
$$


TABLE I

COMPUTATIONAL COMPLEXITY OF ESTIMATORS

\begin{tabular}{|c|c|c|}
\hline Algorithm & Multiplication/Division & Addition/Subtraction \\
\hline RGN & 42 & 21 \\
\hline HMA & 7 & 4 \\
\hline GCMA & 12 & 5 \\
\hline
\end{tabular}

RGN, HMA, and GCMA algorithms at each iteration are listed in Table I. We see that the computational complexity of the HMA and GCMA methods is significantly smaller than that of the original RGN scheme, with the former being the simplest estimator.

\section{Estimation Accuracy Analysis}

In the following, we will show that the covariance matrices for the parameter estimates of a stationary $\boldsymbol{\theta}(n)$, say, $\boldsymbol{\theta}=[A, \phi]^{T}$, in the RGN, HMA, and GCMA algorithms are identical at sufficiently large iteration number.

Starting with the covariance matrix of the RGN algorithm, denoted by $\operatorname{cov}(\hat{\boldsymbol{\theta}}(n))$, it has the form of [6]

$$
\begin{aligned}
\operatorname{cov}(\hat{\boldsymbol{\theta}}(n))= & E\left\{\left[\frac{\partial \mathcal{E}^{2}(n)}{\partial \hat{\boldsymbol{\theta}}^{2}}\right]^{-1}\left[\frac{\partial \mathcal{E}(n)}{\partial \hat{\boldsymbol{\theta}}}\right]\right. \\
& \left.\times\left[\frac{\partial \mathcal{E}(n)}{\partial \hat{\boldsymbol{\theta}}}\right]^{T}\left[\frac{\partial E^{2}(n)}{\partial \hat{\boldsymbol{\theta}}^{2}}\right]^{-1}\right\}\left.\right|_{\hat{\boldsymbol{\theta}}_{(n)=\boldsymbol{\theta}}} \\
= & \sigma^{2}\left[\sum_{i=0}^{n} \lambda^{n-i} \boldsymbol{\psi}(i) \boldsymbol{\psi}^{T}(i)\right]^{-1} \\
& \times \sum_{i=0}^{n} \lambda^{2 n-2 i} \boldsymbol{\psi}(i) \boldsymbol{\psi}^{T}(i) \\
& \times\left[\sum_{i=0}^{n} \lambda^{n-i} \boldsymbol{\psi}(i) \boldsymbol{\psi}^{T}(i)\right]^{-1}
\end{aligned}
$$

where $E$ denotes the expectation operator. When $n$ is sufficiently large, we utilize (9) to get

$$
\operatorname{cov}(\hat{\boldsymbol{\theta}}(n)) \approx \frac{\sigma^{2}(1-\lambda)\left(1+\lambda^{n+1}\right)}{(1+\lambda)\left(1-\lambda^{n+1}\right)}\left[\begin{array}{cc}
2 & 0 \\
0 & 2 / A^{2}
\end{array}\right]
$$

where the variances of $\hat{A}(n)$ and $\hat{\phi}(n)$ are given by the $(1,1)$ and $(2,2)$ entries of $(20)$, respectively. Since both HMA and GCMA approximate the Hessian matrix, it can be easily seen that their asymptotic covariance matrices are also given by (20). In particular, when $\lambda=1$, (20) becomes

$$
\operatorname{cov}(\hat{\boldsymbol{\theta}}(n))=\frac{\sigma^{2}}{n+1}\left[\begin{array}{cc}
2 & 0 \\
0 & 2 / A^{2}
\end{array}\right] .
$$

The mean-square errors of $\hat{A}(n)$ and $\hat{\phi}(n)$ are $2 \sigma^{2} /(n+1)$ and $2 \sigma^{2} /\left((n+1) A^{2}\right)$, which attain their corresponding Cramér-Rao lower bounds (CRLBs), when $n$ is large enough and $v(n)$ is Gaussian distributed. As a result, it is proved that the RGN, HMA, and GCMA algorithms achieve optimal performance for stationary amplitude and phase estimation in an asymptotic sense with $\lambda=1$.

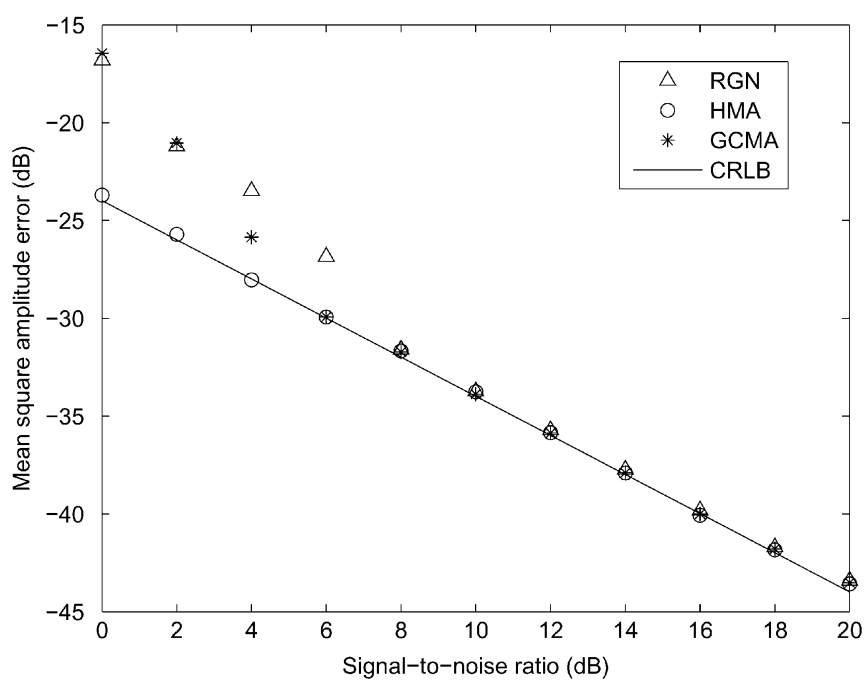

Fig. 1. Mean-square amplitude error versus SNR at stationary condition.

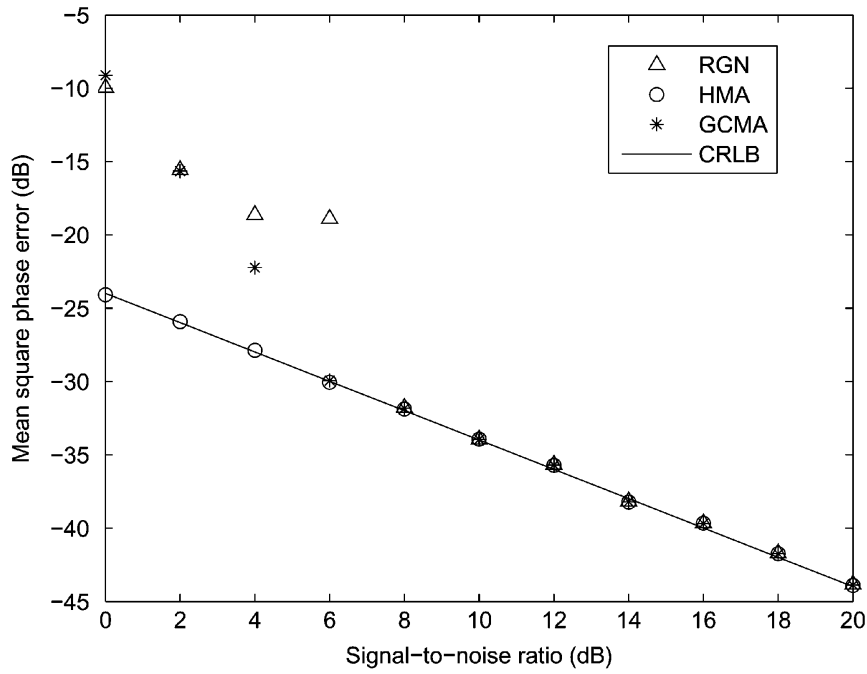

Fig. 2. Mean-square phase error versus SNR at stationary condition.

\section{Simulation Results}

Computer simulations have been carried out to evaluate the performance of the proposed HMA and GCMA methods in amplitude and phase estimation of a noisy sinusoid. The sinusoidal frequency $\omega$ is fixed to be $\pi / 3$, and $v(n)$ is a white Gaussian noise. The estimate of $\boldsymbol{\theta}$ is initialized with $\hat{\boldsymbol{\theta}}(0)=[0.8,0.8]^{T}$ and the observation data length of 500. All simulation results are averages of 1000 independent runs.

Figs. 1 and 2 plot the mean-square error (MSE) performance for amplitude and phase, respectively, of the proposed algorithms versus signal-to-noise ratio (SNR) when both are stationary, namely, $\boldsymbol{\theta}=[1, \pi / 3]^{T}$. The SNR is defined as $A^{2} /\left(2 \sigma^{2}\right)$, and we scale the noise sequence to obtain different conditions. Comparison with the RGN method as well as CRLB is also made. The forgetting factors in all estimators are set to unity in order to achieve their best performance. From the two figures, we see that all three estimators perform similarly, and their MSEs are close to the corresponding CRLBs for sufficiently large SNRs. In fact, the HMA scheme has the 


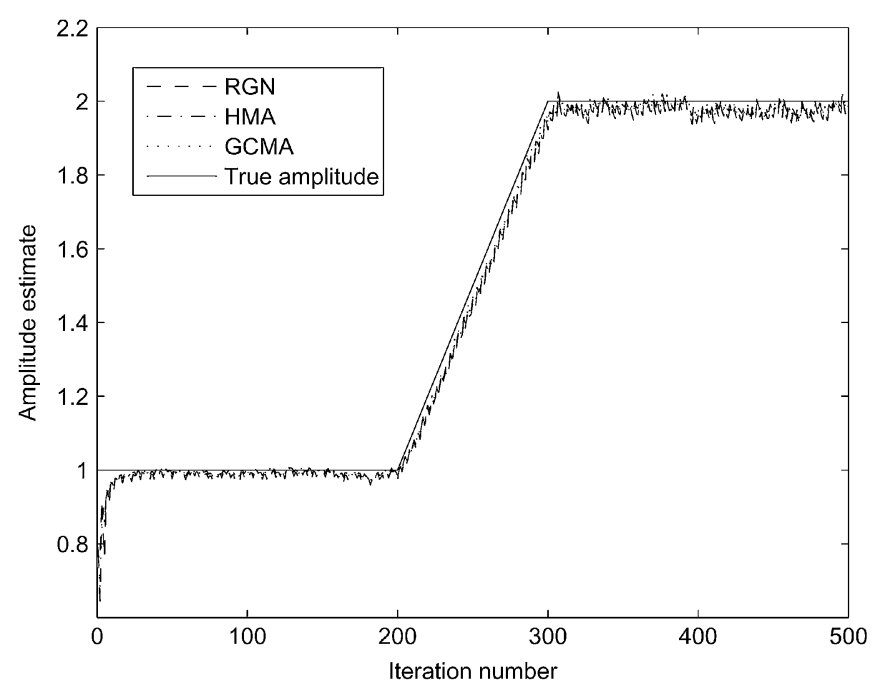

Fig. 3. Amplitude estimate at nonstationary condition.

best threshold performance as it provides optimum estimation performance for the whole SNR range.

In the second test, we examine the estimator performance for time-varying amplitude and phase at $\mathrm{SNR}=10 \mathrm{~dB}$. The amplitude of the sinusoid is set to 1 for the first 200 iterations, then increases linearly at the rate of $A(n)=0.01 n$ for the next 100 iterations, and remains unchanged afterwards. On the other hand, the phase $\phi$ is set to $0.4 \pi$ for the first 200 iterations, then decreases linearly at the rate of $\phi(n)=-0.002 n \pi$ for the next 100 iterations, and remains unchanged afterwards. In this nonstationary scenario, the forgetting factor $\lambda$ is set to 0.8 for fast convergence. Figs. 3 and 4 show the tracking results for the amplitude and phase, respectively, of the HMA, GCMA, and RGN algorithms. It is observed that the proposed methods, which accurately track the time-varying parameters, achieve almost the same performance as that of the RGN method. Although not shown, the MSE results of the three estimators also have very similar values.

\section{CONCLUSION}

Two algorithms have been proposed to simplify the RGN method for direct estimation of amplitude and phase of a noisy

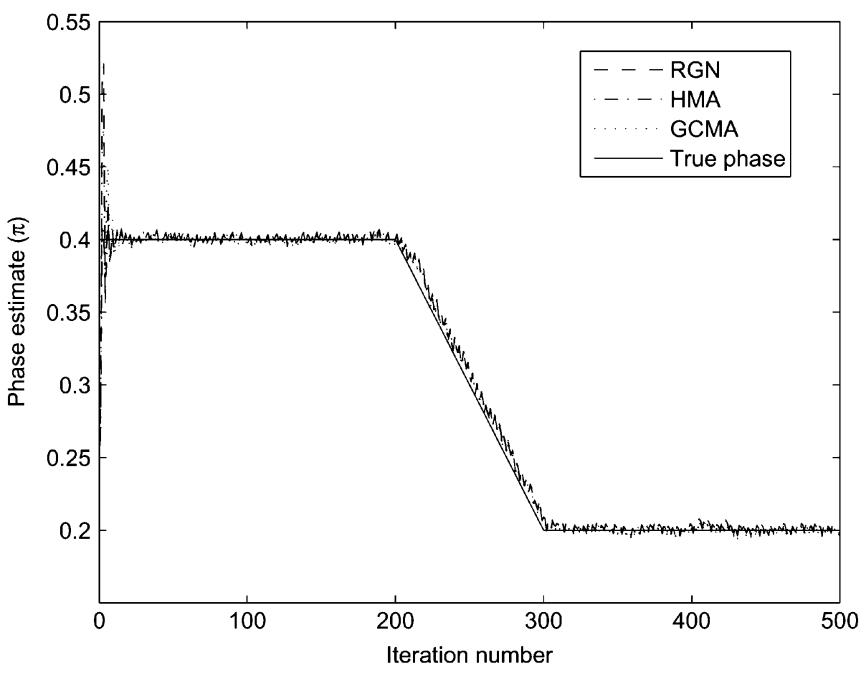

Fig. 4. Phase estimate at nonstationary condition.

sinusoid with known frequency. The first algorithm prunes the Hessian matrix of the RGN scheme directly, while the second approximates the update progress of the Hessian matrix. It is demonstrated that the proposed methods can attain identical tracking performance as the standard RGN algorithm but are much more computationally efficient.

\section{REFERENCES}

[1] S. Kay, Fundamentals of Statistical Signal Processing: Estimation Theory. Englewood Cliffs, NJ: Prentice-Hall, 1993.

[2] P. Stoica, H. Li, and J. Li, "Amplitude estimation of sinusoidal signals: Survey, new results, and an application," IEEE Trans. Signal Process., vol. 48, no. 2, pp. 338-352, Feb. 2000

[3] P. Stoica, R. Moses, B. Friedlander, and T. Soderstrom, "Maximum likelihood estimation of the parameters of multiple sinusoids from noisy measurements," IEEE Trans. Acoust., Speech, Signal Process., vol. ASSP-37, no. 3, pp. 378-392, Mar. 1989.

[4] K. Peters and S. Kay, "Unbiased estimation of the phase of a sinusoid," in Proc. ICASSP, Montreal, QC, Canada, May 2004, vol. 2, pp. 493-496.

[5] H. Fu and P. Kam, "MAP/ML estimation of the frequency and phase of a single sinusoid in noise," IEEE Trans. Signal Process., vol. 55, no. 3, pp. 834-845, Mar. 2007.

[6] L. Ljung and T. Soderstrom, Theory and Practice of Recursive Identification. Cambridge, MA: MIT Press, 1987. 